\title{
SIMULTANEOUS BAYESIAN INFERENCE FOR LONGITUDINAL DATA WITH ASYMMETRY, LEFT-CENSORING AND COVARIATES MEASURED WITH ERRORS
}

\author{
Yangxin Huang* and Getachew Dagne*
}

\begin{abstract}
It is a common practice to analyze complex longitudinal data using flexible nonlinear mixed-effects (NLME) models with normality assumption. However, a serious departure of normality may cause lack of robustness and subsequently lead to invalid inference and unreasonable estimates. Covariates are usually introduced in such models to partially explain inter-subject variations, but some covariates may be often measured with substantial errors. Moreover, the response observations may be subject to left-censoring due to a detection limit. Inferential procedures can be complicated dramatically when data with asymmetric (skewed) characteristics, leftcensoring and measurement errors are observed. In the literature, there has been considerable interest in accommodating either skewness, censoring or covariate measurement errors in such models, but there is relatively little work concerning all of the three features simultaneously. In this article, we jointly investigate a skew- $t$ NLME model for response (with left-censoring) process and a skew- $t$ nonparametric mixedeffects model for covariate (with measurement errors) process. We propose a robust skew- $t$ Bayesian modeling approach in a general form to analyze data in capturing the effects of skewness, censoring and measurement errors in covariates simultaneously. A real data example is offered to illustrate the methodologies. The proposed modeling alternative offers important advantages in the sense that the model can be easily fitted in freely available software and the computational effort for the model with a skew- $t$ distribution is almost equivalent to that of the model with a standard normal distribution.
\end{abstract}

Key words and phrases: Bayesian analysis, covariate measurement errors, HIV and AIDS clinical trials, limit of detection, mixed-effects models, skew distributions.

\section{Introduction}

Longitudinal data analysis has attracted a considerable research interest and a large number of statistical modeling and analysis methods that have been suggested for analyzing such data with various features. However, there is relatively little work done on simultaneously accounting for skewness, left-censoring due to a limit of detection (i.e., a certain threshold below which viral loads are not quantifiable) and covariate measurement error, which are inherent features of longitudinal data. Firstly, the commonly assumed distribution for model random errors is normal, but such an assumption may lack the robustness against departures from normality and/or outliers and may obscure important features

Received May 23, 2010. Revised January 17, 2012. Accepted April 29, 2012.

*Department of Epidemiology \& Biostatistics, College of Public Health, University of South Florida, Tampa, FL 33612, U.S.A. Email: yhuang@health.usf.edu 
of between- and within-subject variations since collected data are often far from symmetric behavior. Thus statistical inference and analysis with normal assumption may lead to biased or misleading results (Huang and Dagne (2011), Verbeke and Lesaffre (1996), Sahu et al. (2003)). Specially non-normal characteristics such as skewness with heavy right or left tail appear often in virologic responses. For example, Fig. 1 (top panel) displays the histograms of repeated viral load (in ln scale) and CD4 cell count measurements for 44 subjects enrolled in an AIDS clinical study (A5055) (Acosta et al. (2004)). It is seen that, for this data set to be analyzed in this paper, the both viral load (even after ln-transformation) and CD4 cell count are highly skewed, and thus a normality assumption may be violated. Secondly, the validity of inference methods relies on an important requirement that variables are "perfectly" measured. In practice, however, collected data are often far from "perfect". Although longitudinal studies are designed to collect data on every individual in the study at each assessment, the response (viral load) measurements may be subject to left-censoring due to a detection limit. Figure 1 (bottom panel) shows the measurements of viral load and CD4 cell count for three randomly selected patients in A5055 study. We can see that for some patients viral loads are below a limit of detection (LOD, 50 copies $/ \mathrm{mL}$ here). When response observations are below LOD, a common practice is to impute the censored values by the detection limit or half the detection limit (Ding and Wu (1999), Davidian and Giltinan (1995), Wu (2002)). Such ad hoc methods may produce biased results (Hughes (1999)). Thirdly, the nonlinear mixed-effects (NLME) models have been used in the previous studies to account for both between-subject and within-subject variations in response which is associated with covariates including CD4 cell count. However, the covariates such as CD4 cell count are often measured with substantial errors. Thus measurement error in covariates is another typical feature of longitudinal data and statistical inference without considering measurement errors in covariates may result in biased results (Huang and Dagne (2011), Liu and Wu (2007), Wu (2002)).

Our research was motivated by the AIDS clinical trial considered by Acosta et al. (2004). In this study, $44 \mathrm{HIV}-1$ infected patients were treated with a potent artiretroviral regimen. RNA viral load was measured in copies $/ \mathrm{mL}$ at study days $0,7,14,28,56,84,112,140$ and 168 of follow-up. Covariates such as CD4 cell counts were also measured throughout the study on similar scheme. In this study, the viral load detectable limit is 50 copies $/ \mathrm{mL}$, and there are 54 out of 357 (15 percent) of all viral load measurements that are below the detection limit. Previous studies shew that change in viral load may be associated with change in CD4 cell counts. It is important to study the patterns of virological response to treatment in order to make clinical decisions and provide individualized treatments. Since viral load measurements appear to be skewed and censored, and in addition CD4 cell counts are typically measured with substantial errors and skewness, statistical analyses must take all these factors into account.

Much of the statistical literature on modeling of longitudinal data has fo- 
(a):Histogram of viral load in In scale

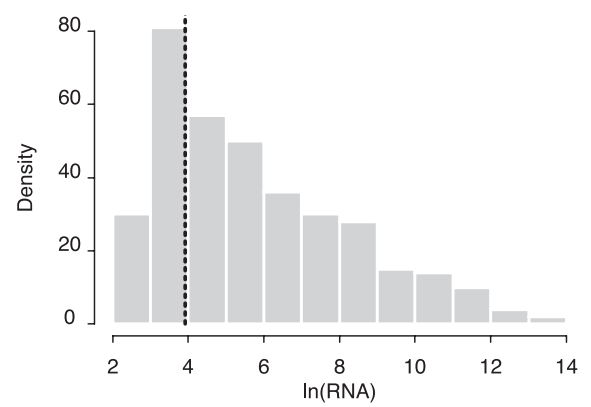

(c): Profiles of viral load in In scale

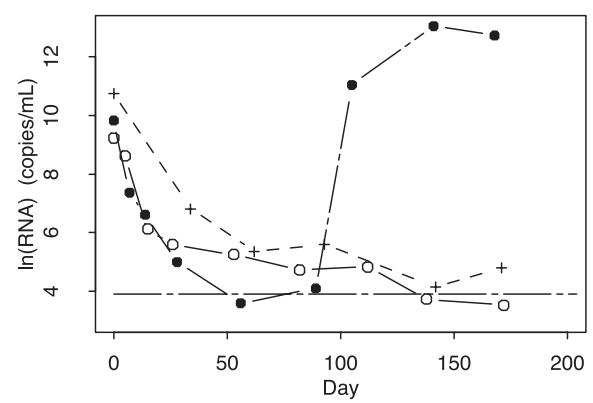

(b):Histogram of CD4 cell count

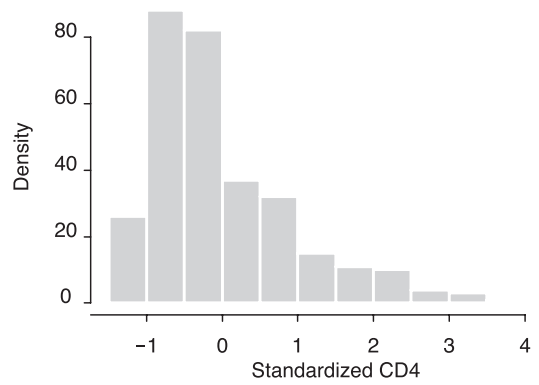

(d): Profiles of CD4 cell count

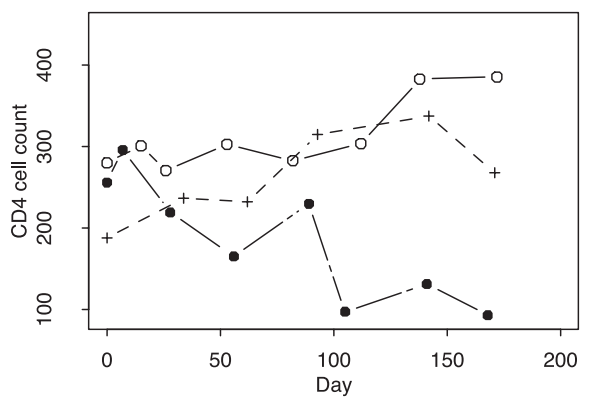

Figure 1. The histograms (top panel) of viral load measured from RNA levels (in natural $\mathrm{ln}$ scale) and standardized CD4 cell count in plasma for 44 patients in an AIDS clinical trial study. Profiles (bottom panel) of viral load (response) in ln scale and CD4 cell count (covariate) for three randomly selected patients. The vertical and horizontal lines in (a) and (c) are below the detectable level of viral load $(3.91=\ln (50))$.

cused on the development of models that aim at capturing only specific aspects of motivating case studies. These include, but are not limited to, a linear mixedeffects (LME) model with a mixture of normal distributions (Verbeke and Lesaffre (1996)); a skew-normal (SN) nonlinear mixed-effects (NLME) model for longitudinal data with skewness and mismeasured covariates (Huang and Dagne (2011)); and an NLME model with a normal distribution for longitudinal data with censoring and covariate measurement errors ( $\mathrm{Wu}$ (2002)). However, relatively few studies have been conducted on simultaneously accounting for the biases induced by asymmetry, incompleteness, and mismeasured covariates. It is not clear how asymmetry, left-censoring due to LOD, and covariate measurement error of data may interact and simultaneously influence inferential procedures. Statistical inference and analysis could be complicated dramatically when these three features arise. The goal of this article is to investigate the effects on inference when all of the three typical features exist in the longitudinal data. To achieve our objective we employ a fairly general framework to accommodate a large class of problems with various features. Specifically, a robust Bayesian inference approach is employed to jointly investigate the NLME model with a skew-t (ST) distribution 
for the response process in the presence of left-censoring, and the nonparametric mixed-effects model with $\mathrm{ST}$ distribution for covariate measurement error process. Estimation of model parameters is carried out using a simultaneous Bayesian inference approach via MCMC algorithm. We consider a multivariate ST distribution introduced by Sahu et al. (2003) which are suitable for a Bayesian inference and are briefly discussed in Appendix. In Section 2, we present the joint models with ST distribution and associated Bayesian modeling approach in general forms so that they can be applicable to other scientific fields. In Section 3, we discuss specific joint models for HIV response process with left-censoring and CD4 covariate process with measurement error that are used to illustrate the proposed methods using the data set described above and report the analysis results. Finally, we conclude paper with discussion in Section 4.

\section{Joint models and associated inferential methods}

\subsection{Skew- $t$ joint models}

In this section, we present the models and methods in a general form, illustrating that our methods may be applicable in other applications. Denote the number of subjects by $n$ and the number of measurements on the $i$ th subject by $n_{i}$. Let $y_{i j}$ be the value of response for the individual $i$ at time $t_{i j}$ $\left(i=1,2, \ldots, n ; j=1,2, \ldots, n_{i}\right)$. The observed value $y_{i j}$ is $\left(q_{i j} ; c_{i j}\right)$, where $c_{i j}$ is the censoring indicator such that $y_{i j}$ is observed if $c_{i j}=0$ and $y_{i j}$ is left censored if $c_{i j}=1$, i.e. $y_{i j}=q_{i j}$ if $c_{i j}=0$, and $y_{i j} \leq d$ if $c_{i j}=1$, where $d$ is some known constant (e.g. LOD). For simplicity, we consider a single timevarying covariate $z_{i j}$ for the individual $i$ at time $t_{i j}$. Let the observed data $\Re=\left\{\left(\boldsymbol{q}_{i}, \boldsymbol{c}_{i}, \boldsymbol{z}_{i}\right), i=1, \ldots, n\right\}$, where $\boldsymbol{y}_{i}=\left(y_{i 1}, \ldots, y_{i n_{i}}\right)^{T}, \boldsymbol{z}_{i}=\left(z_{i 1}, \ldots, z_{i n_{i}}\right)^{T}$, $\boldsymbol{q}_{i}=\left(q_{i 1}, \ldots, q_{i n_{i}}\right)^{T}$ and $\boldsymbol{c}_{i}=\left(c_{i 1}, \ldots, c_{i n_{i}}\right)^{T}$.

Various covariate models were investigated in the literature (Carroll et al. (2006), Higgins et al. (1997), Liu and Wu (2007), Wu (2002)). However, the fundamental assumption of distributions for model random error is usually normal in the previous studies and this assumption may lack the robustness against departures from normality and/or may violate the agreement with observed data. Thus statistical inference and analysis with normal assumption may lead to misleading results. In this paper, we extend the covariate models by considering model random error with an ST distribution. In the presence of measurement errors in covariate, we adopt a flexible empirical nonparametric mixed-effects model with an ST to quantify the covariate process as follows.

$$
\begin{aligned}
& z_{i j}=w\left(t_{i j}\right)+h_{i}\left(t_{i j}\right)+\epsilon_{i j}\left(\equiv z_{i j}^{*}+\epsilon_{i j}\right) \\
& \boldsymbol{\epsilon}_{i} \text { iid } \sim S T_{n_{i}, \nu_{\epsilon}}\left(\mathbf{0}, \tau^{2} \boldsymbol{I}_{n_{i}}, \boldsymbol{\Delta}\left(\boldsymbol{\delta}_{\epsilon_{i}}\right)\right),
\end{aligned}
$$

where $w\left(t_{i j}\right)$ and $h_{i}\left(t_{i j}\right)$ are unknown nonparametric smooth fixed-effects and random-effects functions, respectively, and $\boldsymbol{\epsilon}_{i}=\left(\epsilon_{i 1}, \ldots, \epsilon_{i n_{i}}\right)^{T}$ follows a multivariate ST distribution with the degree of freedom $\nu_{\epsilon}$, the unknown precision parameter $\tau^{2}$ and the $n_{i} \times n_{i}$ skewness diagonal matrix $\boldsymbol{\Delta}\left(\boldsymbol{\delta}_{\epsilon_{i}}\right)=\operatorname{diag}\left(\delta_{\epsilon_{i 1}}, \ldots, \delta_{\epsilon_{i n_{i}}}\right)$ with $n_{i} \times 1$ skewness parameter vector $\delta_{\epsilon_{i}}=\left(\delta_{\epsilon_{i 1}}, \ldots, \delta_{\epsilon_{i n_{i}}}\right)^{T}$. In particular, if 
$\delta_{\epsilon_{i 1}}=\cdots=\delta_{\epsilon_{i n_{i}}} \hat{=} \delta_{\epsilon}$, then $\boldsymbol{\Delta}\left(\boldsymbol{\delta}_{\epsilon_{i}}\right)=\delta_{\epsilon} \boldsymbol{I}_{n_{i}}$ and $\boldsymbol{\delta}_{\epsilon_{i}}=\delta_{\epsilon} \mathbf{1}_{n_{i}}$ with $\mathbf{1}_{n_{i}}=(1, \ldots, 1)^{T}$; this indicates that we are interested in skewness of overall data set and is the case to be used in real data analysis in Section $3 . z_{i j}^{*}=w\left(t_{i j}\right)+h_{i}\left(t_{i j}\right)$ are the true but unobservable covariate values at time $t_{i j}$. The fixed smooth function $w(t)$ represents population average of the covariate process, while the random smooth function $h_{i}(t)$ is introduced to incorporate the large inter-individual variation in the covariate process. We assume that $h_{i}(t)$ is the realization of a zero-mean stochastic process.

Nonparametric mixed-effects model (2.1) is more flexible than parametric mixed-effects models. To fit model (2.1), we apply a regression spline method to $w(t)$ and $h_{i}(t)$. The working principle is briefly described as follows and more details can be found in literature (Wu and Zhang (2002), Davidian and Giltinan (1995)). The main idea of regression spline is to approximate $w(t)$ and $h_{i}(t)$ by using a linear combination of spline basis functions. For instance, $w(t)$ and $h_{i}(t)$ can be approximated by a linear combination of basis functions $\boldsymbol{\Psi}_{p}(t)=\left\{\psi_{0}(t), \psi_{1}(t), \ldots, \psi_{p-1}(t)\right\}^{T}$ and $\boldsymbol{\Phi}_{q}(t)=\left\{\phi_{0}(t), \phi_{1}(t), \ldots, \phi_{q-1}(t)\right\}^{T}$, respectively. That is,

$$
\begin{aligned}
& w(t) \approx w_{p}(t)=\sum_{l=0}^{p-1} \alpha_{l} \psi_{l}(t)=\boldsymbol{\Psi}_{p}(t)^{T} \boldsymbol{\alpha}, \\
& h_{i}(t) \approx h_{i q}(t)=\sum_{l=0}^{q-1} \alpha_{i l} \phi_{l}(t)=\boldsymbol{\Phi}_{q}(t)^{T} \boldsymbol{a}_{i},
\end{aligned}
$$

where $\boldsymbol{\alpha}=\left(\alpha_{0}, \ldots, \alpha_{p-1}\right)^{T}$ is a $p \times 1$ vector of fixed-effects and $\boldsymbol{a}_{i}=\left(a_{i 0}, \ldots\right.$, $\left.a_{i, q-1}\right)^{T}(q \leq p)$ is a $q \times 1$ vector of random-effects with $\boldsymbol{a}_{i}$ iid $\sim N_{q}\left(\mathbf{0}, \boldsymbol{\Sigma}_{a}\right)$. Based on the assumption of $h_{i}(t)$, we can regard $\boldsymbol{a}_{i}$ as iid realizations of a zeromean random vector. For our model, we consider natural cubic spline bases with the percentile-based knots. To select an optimal degree of regression spline and numbers of knots, i.e., optimal sizes of $p$ and $q$, the Akaike information criterion (AIC) or the Bayesian information criterion (BIC) is often applied (Wu and Zhang, 2002; Davidian and Giltinan (1995)).

Substituting $w(t)$ and $h_{i}(t)$ by their approximations $w_{p}(t)$ and $h_{i q}(t)$, we can approximate model (2.1) by the following linear mixed-effects (LME) model.

$$
\begin{aligned}
& z_{i j} \approx \boldsymbol{\Psi}_{p}\left(t_{i j}\right)^{T} \boldsymbol{\alpha}+\mathbf{\Phi}_{q}\left(t_{i j}\right)^{T} \boldsymbol{a}_{i}+\epsilon_{i j} \approx z_{i j}^{*}+\epsilon_{i j}, \\
& \boldsymbol{\epsilon}_{i} \text { iid } \sim S T_{n_{i}, \nu_{\epsilon}}\left(\mathbf{0}, \tau^{2} \boldsymbol{I}_{n_{i}}, \boldsymbol{\Delta}\left(\boldsymbol{\delta}_{\epsilon_{i}}\right)\right),
\end{aligned}
$$

For the response process with left-censoring, we consider the following NLME model with an ST distribution, which incorporates possibly mismeasured timevarying covariates

$$
\begin{aligned}
& y_{i j}=g\left(t_{i j}, \boldsymbol{\beta}_{i j}\right)+e_{i j}, \quad \boldsymbol{e}_{i} \text { iid } \sim S T_{n_{i}, \nu_{e}}\left(\mathbf{0}, \sigma^{2} \boldsymbol{I}_{n_{i}}, \boldsymbol{\Delta}\left(\boldsymbol{\delta}_{e_{i}}\right)\right), \\
& \boldsymbol{\beta}_{i j}=\boldsymbol{d}\left(z_{i j}^{*}, \boldsymbol{\beta}, \boldsymbol{b}_{i}\right), \quad \boldsymbol{b}_{i} \text { iid } \sim N_{s_{3}}\left(\mathbf{0}, \boldsymbol{\Sigma}_{b}\right),
\end{aligned}
$$


where $g(\cdot)$ is a nonlinear known function, $\boldsymbol{d}(\cdot)$ is an $s_{1}$-dimensional vectorvalued linear function, $\boldsymbol{\beta}_{i j}$ are $s_{1} \times 1$ individual-specific time-dependent parameter vectors, $\boldsymbol{\beta}$ is $s_{2} \times 1$ population parameter vector $\left(s_{2} \geq s_{1}\right), \boldsymbol{b}_{i}$ are $s_{3} \times 1$ vector of random-effects $\left(s_{3} \leq s_{1}\right)$ and $\boldsymbol{\Sigma}_{b}$ is the unrestricted covariance matrix, $\boldsymbol{e}_{i}=\left(e_{i 1}, \ldots, e_{i n_{i}}\right)^{T}$ follows a multivariate ST distribution with degree of freedom $\nu_{e}$, unknown precision parameter $\sigma^{2}$ and $n_{i} \times n_{i}$ skewness diagonal matrix $\boldsymbol{\Delta}\left(\boldsymbol{\delta}_{e_{i}}\right)=\operatorname{diag}\left(\delta_{e_{i 1}}, \ldots, \delta_{e_{i n_{i}}}\right)$ with $n_{i} \times 1$ skewness parameter vector $\delta_{e_{i}}=\left(\delta_{e_{i 1}}, \ldots, \delta_{e_{i n_{i}}}\right)^{T}$. In particular, if $\delta_{e_{i 1}}=\cdots=\delta_{e_{i_{i}}} \hat{=} \delta_{e}$, then $\boldsymbol{\Delta}\left(\boldsymbol{\delta}_{e_{i}}\right)=\delta_{e} \boldsymbol{I}_{n_{i}}$ and $\boldsymbol{\delta}_{e_{i}}=\delta_{e} \mathbf{1}_{n_{i}}$. In model (2.4), we assume that the individualspecific parameters $\boldsymbol{\beta}_{i j}$ depend on the true (but unobservable) covariate $z_{i j}^{*}$ rather than the observed covariate $z_{i j}$, which may be measured with errors. In the response model (2.4), we link the time-dependent parameters $\boldsymbol{\beta}_{i j}$ to the covariate through $z_{i j}^{*}$ which summarizes the covariate process.

\subsection{Simultaneous Bayesian inference}

In a longitudinal study, such as the AIDS study described previously, the longitudinal response and covariate processes are usually connected physically or biologically. Statistical inference based on the commonly used two-step method may be undesirable since it fails to take the covariate estimation into account (Higgins et al. (1997)). Although a simultaneous inference method based on a joint likelihood for the covariate and response data may be favorable, the computation associated with the joint likelihood inference in joint models of longitudinal data can be extremely intensive and may lead to convergence problems, and in some cases it can even be computationally infeasible (Wu (2002), Liu and $\mathrm{Wu}$ (2007)). Here we propose a simultaneous Bayesian inference method based on MCMC procedure for longitudinal data of response with left-censoring and covariate with measurement error. The Bayesian joint modeling approach may pave a way to alleviate the computational burdens and to overcome convergence problems.

We assume that $\boldsymbol{a}_{i}, \boldsymbol{b}_{i}, \boldsymbol{\epsilon}_{i}$ and $\boldsymbol{e}_{i}$ are mutually independent of each other. Following Sahu et al. (2003) and properties of ST distribution, in order to specify models (2.3) and (2.4) for MCMC computation it can be shown by introducing two random variable vectors $\boldsymbol{w}_{e_{i}}=\left(w_{e_{i 1}}, \ldots, w_{e_{i n_{i}}}\right)^{T}$ and $\boldsymbol{w}_{\epsilon_{i}}=\left(w_{\epsilon_{i 1}}, \ldots, w_{\epsilon_{i n_{i}}}\right)^{T}$ and two random variables $u_{\epsilon_{i}}$ and $u_{e_{i}}(i=1, \ldots, n)$ based on the stochastic representation for the ST distribution (see Appendix for details) that $z_{i j}$ and $y_{i j}$ can be hierarchically formulated as

$$
\begin{aligned}
& z_{i j} \mid \boldsymbol{a}_{i}, w_{\epsilon_{i j}}, u_{\epsilon_{i}} ; \boldsymbol{\alpha}, \tau^{2}, \delta_{\epsilon_{i j}} \sim N\left(z_{i j}^{*}+\delta_{\epsilon_{i j}} w_{\epsilon_{i j}}, \frac{\tau^{2}}{u_{\epsilon_{i}}}\right), \\
& w_{\epsilon_{i j}} \sim N(0,1) I\left(w_{\epsilon_{i j}}>0\right), \quad u_{\epsilon_{i}} \mid \nu_{\epsilon} \sim \Gamma\left(\nu_{\epsilon} / 2, \nu_{\epsilon} / 2\right), \\
& y_{i j} \mid \boldsymbol{b}_{i}, w_{e_{i j}}, u_{e_{i}} ; \boldsymbol{\beta}, \sigma^{2}, \delta_{e_{i j}} \sim N\left(g\left(t_{i j}, \boldsymbol{d}\left(z_{i j}^{*}, \boldsymbol{\beta}, \boldsymbol{b}_{i}\right)\right)+\delta_{e_{i j}} w_{e_{i j}}, \frac{\sigma^{2}}{u_{e_{i}}}\right), \\
& w_{e_{i j}} \sim N(0,1) I\left(w_{e_{i j}}>0\right), \quad u_{e_{i}} \mid \nu_{e} \sim \Gamma\left(\nu_{e} / 2, \nu_{e} / 2\right), \\
& \boldsymbol{a}_{i}\left|\boldsymbol{\Sigma}_{a} \sim N_{q}\left(\mathbf{0}, \boldsymbol{\Sigma}_{a}\right), \quad \boldsymbol{b}_{i}\right| \boldsymbol{\Sigma}_{b} \sim N_{s_{3}}\left(\mathbf{0}, \boldsymbol{\Sigma}_{b}\right),
\end{aligned}
$$


where $\Gamma(\cdot)$ is a Gamma distribution, $I\left(w_{e_{i j}}>0\right)$ is an indicator function and $w_{e_{i j}} \sim N(0,1)$ truncated in the space $w_{e_{i j}}>0$ (i.e., the standard half-normal distribution); $w_{\epsilon_{i j}}$ can be defined similarly. $z_{i j}^{*}$ is viewed as the true but unobservable covariate values at time $t_{i j}$. It is noted that as discussed in Appendix, the hierarchical model with the ST distribution (2.5) is reduced to the following three special cases: (i) a model with skew-normal (SN) distribution as $\nu_{\epsilon}, \nu_{e} \rightarrow \infty$ and $u_{\epsilon_{i}}, u_{e_{i}} \rightarrow 1$ with probability 1 (i.e., the two corresponding distributional specifications are omitted in (2.5)); (ii) a model with standard $t$-distribution as $\delta_{\epsilon_{i j}}=\delta_{e_{i j}}=0$, and thus the two distributional specifications of $w_{\epsilon_{i j}}$ and $w_{e_{i j}}$ are omitted in (2.5); and (iii) a model with standard normal distribution as $\nu_{\epsilon}, \nu_{e} \rightarrow \infty$ and $\delta_{\epsilon_{i j}}=\delta_{e_{i j}}=0$; in this case, the four corresponding distributional specifications of $w_{\epsilon_{i j}}, w_{e_{i j}}, u_{\epsilon_{i}}$ and $u_{e_{i}}$ are omitted in (2.5).

Under Bayesian framework, we still need to specify prior distributions for unknown parameters in models (2.3) and (2.4) as follows.

$$
\begin{array}{ll}
\boldsymbol{\alpha} \sim N_{p}\left(\boldsymbol{\alpha}_{0}, \boldsymbol{\Lambda}_{1}\right), & \tau^{2} \sim I G\left(\omega_{1}, \omega_{2}\right), \\
\boldsymbol{\Sigma}_{a} \sim I W\left(\boldsymbol{\Omega}_{1}, \rho_{1}\right), & \boldsymbol{\delta}_{\epsilon_{i}} \sim N_{n_{i}}\left(\mathbf{0}, \boldsymbol{\Gamma}_{1}\right), \\
\boldsymbol{\beta} \sim N_{s_{2}}\left(\boldsymbol{\beta}_{0}, \boldsymbol{\Lambda}_{2}\right), & \sigma^{2} \sim I G\left(\omega_{3}, \omega_{4}\right), \\
\boldsymbol{\Sigma}_{b} \sim I W\left(\boldsymbol{\Omega}_{2}, \rho_{2}\right), & \boldsymbol{\delta}_{e_{i}} \sim N_{n_{i}}\left(\mathbf{0}, \boldsymbol{\Gamma}_{2}\right), \\
\nu_{\epsilon} \sim \operatorname{Exp}\left(\nu_{10}\right) I\left(\nu_{\epsilon}>3\right), \quad \nu_{e} \sim \operatorname{Exp}\left(\nu_{20}\right) I\left(\nu_{e}>3\right),
\end{array}
$$

where the mutually independent Inverse Gamma $(I G)$, Normal $(N)$, Exponential $(E x p)$ and Inverse Wishart ( $I W)$ prior distributions are chosen to facilitate computations (Davidian and Giltinan (1995)). The super-parameter matrices $\boldsymbol{\Lambda}_{1}$, $\boldsymbol{\Lambda}_{2}, \boldsymbol{\Omega}_{1}, \boldsymbol{\Omega}_{2}, \boldsymbol{\Gamma}_{1}$ and $\boldsymbol{\Gamma}_{2}$ can be assumed to be diagonal for convenient implementation.

Let $f(\cdot \mid \cdot), F(\cdot \mid \cdot)$ and $\pi(\cdot)$ denote a probability density function (pdf), cumulative density function (cdf) and prior density function, respectively. Conditional on the random variables and some unknown parameters, a detectable measurement $y_{i j}$ contributes $f\left(y_{i j} \mid \boldsymbol{b}_{i}, w_{e_{i j}}, u_{e_{i}} ; \boldsymbol{\beta}, \sigma^{2}, \delta_{e_{i j}}\right)$, whereas a non-detectable measurement contributes $F\left(d \mid \boldsymbol{b}_{i}, w_{e_{i j}}, u_{e_{i}} ; \boldsymbol{\beta}, \sigma^{2}, \delta_{e_{i j}}\right) \equiv P\left(y_{i j}<d \mid \boldsymbol{b}_{i}, w_{e_{i j}}, u_{e_{i}}\right.$; $\left.\boldsymbol{\beta}, \sigma^{2}, \delta_{e_{i j}}\right)$ in the likelihood. Let $\boldsymbol{\theta}=\left\{\boldsymbol{\alpha}, \boldsymbol{\beta}, \tau^{2}, \sigma^{2}, \boldsymbol{\Sigma}_{a}, \boldsymbol{\Sigma}_{b}, \nu_{\epsilon}, \nu_{e}, \boldsymbol{\delta}_{\epsilon_{i}}, \boldsymbol{\delta}_{e_{i}} ; i=\right.$ $1, \ldots, n\}$ be the collection of unknown parameters in models (2.3) and (2.4). We assume that $\boldsymbol{\alpha}, \boldsymbol{\beta}, \tau^{2}, \sigma^{2}, \boldsymbol{\Sigma}_{a}, \boldsymbol{\Sigma}_{b}, \nu_{\epsilon}, \nu_{e}, \boldsymbol{\delta}_{\epsilon_{i}}, \boldsymbol{\delta}_{e_{i}}(i=1, \ldots, n)$ are independent of each other, i.e., $\pi(\boldsymbol{\theta})=\pi(\boldsymbol{\alpha}) \pi(\boldsymbol{\beta}) \pi\left(\tau^{2}\right) \pi\left(\sigma^{2}\right) \pi\left(\boldsymbol{\Sigma}_{a}\right) \pi\left(\boldsymbol{\Sigma}_{b}\right) \pi\left(\nu_{\epsilon}\right) \pi\left(\nu_{e}\right) \prod_{i}$. $\pi\left(\boldsymbol{\delta}_{\epsilon_{i}}\right) \pi\left(\boldsymbol{\delta}_{e_{i}}\right)$. After we specify the models for the observed data and the prior distributions for the unknown model parameters, we can make statistical inference for the parameters based on their posterior distributions under Bayesian framework. The joint posterior density of $\boldsymbol{\theta}$ based on the observed data $\Re$ can be given by

$$
f(\boldsymbol{\theta} \mid \Re) \propto\left\{\prod_{i=1}^{n} \iint L_{\boldsymbol{y}_{i}} L_{z_{i}} f\left(\boldsymbol{a}_{i} \mid \boldsymbol{\Sigma}_{a}\right) f\left(\boldsymbol{b}_{i} \mid \boldsymbol{\Sigma}_{b}\right) d \boldsymbol{a}_{i} d \boldsymbol{b}_{i}\right\} \pi(\boldsymbol{\theta}),
$$


where $L_{\boldsymbol{y}_{i}}=\prod_{j=1}^{n_{i}} f\left(y_{i j} \mid \boldsymbol{b}_{i}, w_{e_{i j}}, u_{e_{i}} ; \boldsymbol{\beta}, \sigma^{2}, \delta_{e_{i j}}\right)^{1-c_{i j}} F\left(d \mid \boldsymbol{b}_{i}, w_{e_{i j}}, u_{e_{i}} ; \boldsymbol{\beta}, \sigma^{2}\right.$, $\left.\delta_{e_{i j}}\right)^{c_{i j}} f\left(w_{e_{i j}} \mid w_{e_{i j}}>0\right) f\left(u_{e_{i}} \mid \nu_{e}\right)$ is the likelihood for the observed data $\left\{\left(\boldsymbol{q}_{i}, \boldsymbol{c}_{i}\right), i=1, \ldots, n\right\}$ and $L_{z_{i}}=\prod_{j=1}^{n_{i}} f\left(z_{i j} \mid \boldsymbol{a}_{i}, w_{\epsilon_{i j}}, u_{\epsilon_{i}} ; \boldsymbol{\alpha}, \tau^{2}, \delta_{\epsilon_{i j}}\right) f\left(w_{\epsilon_{i j}} \mid\right.$ $\left.w_{\epsilon_{i j}}>0\right) f\left(u_{\epsilon_{i}} \mid \nu_{\epsilon}\right)$ is the likelihood for the observed data $\left\{z_{i}, i=1, \ldots, n\right\}$. In general, the integrals in (2.7) are of high dimension and do not have closed form. Analytic approximations to the integrals may not be sufficiently accurate. Therefore, it is prohibitive to directly calculate the posterior distribution of $\boldsymbol{\theta}$ based on the observed data. As an alternative, MCMC procedures can be used to sample based on (2.7) using the Gibbs sampler along with the MetropolisHastings (M-H) algorithm. An important advantage of the above representations is that they easy implementation of the methods using the freely-available WinBUGS software (Lunn et al. (2000)) and that the computational effort for the model with an ST distribution is almost equivalent to that of the model with a normal distribution. Note that when WinBUGS software is used to implement our modeling approach, it is not necessary to explicitly specify the full conditional distributions for parameters to be estimated. Although their derivations are straightforward by working the complete joint posterior, some cumbersome algebra will be involved. Thus we omit those here.

\section{Analysis of AIDS data}

\subsection{Specific dynamic models}

We now analyse the data set described in Section 1 based on the proposed the models and methods. Among the 44 eligible patients, the number of viral load measurements for each patient varies from 4 to 9 measurements. As is evident from Fig. 1 (bottom panel), the inter-patient variations in viral load appear to be large and these variations appear to change over time. Previous studies suggest that the inter-patient variation in viral load may be partially explained by timevarying CD4 cell count (Huang et al. (2006), Wu (2002)).

Models for the covariate process are needed in order to incorporate measurement errors in covariates. CD4 measures are known with nonnegligible errors and ignoring covariate measurement errors can lead to severely misleading results in a statistical inference (Carroll et al. (2006)). In A5055 study, roughly 10 per cent of the CD4 measurement times are inconsistent with the viral load measurement times. Thus CD4 measurements may be missed at viral load measurement times due mainly to a different CD4 measurement scheme as designed in the study (for example, CD4 measurements were missed at day 7 displayed in Fig. 1 (bottom panel). There seem to be no particular patterns for the missingness. Thus we assume that the missing data in CD4 are missing at random (MAR) in the sense of Rubin (1976), so that the missing data mechanism can be ignored in the analysis. With CD4 measures collected over time from the AIDS study, we may model the CD4 process to partially address the measurement errors (Wu (2002)). However, the CD4 trajectories are often complicated, and there is no well established model for the CD4 process. We, thus, model the CD4 process empirically using a nonparametric mixed-effects model, which is flexible and works well for com- 
plex longitudinal data. We use linear combinations of natural cubic splines with percentile-based knots to approximate $w(t)$ and $h_{i}(t)$. Following the study in Liu and $\mathrm{Wu}(2007)$, we set $\psi_{0}(t)=\phi_{0}(t)=1$ and take the same natural cubic splines in the approximations (2.2) with $q \leq p$. The values of $p$ and $q$ are determined based on the AIC/BIC model selection criteria which suggest the following ST nonparametric mixed-effects CD4 covariate model.

$$
z_{i j}=\left(\alpha_{0}+a_{i 0}\right)+\left(\alpha_{1}+a_{i 1}\right) \psi_{1}\left(t_{i j}\right)+\left(\alpha_{2}+a_{i 2}\right) \psi_{2}\left(t_{i j}\right)+\epsilon_{i j}\left(\equiv z_{i j}^{*}+\epsilon_{i j}\right)
$$

where $z_{i j}$ is the observed CD4 value at time $t_{i j}, \psi_{1}(\cdot)$ and $\psi_{2}(\cdot)$ are two basis functions given in Section 2, $\boldsymbol{\alpha}=\left(\alpha_{0}, \alpha_{1}, \alpha_{2}\right)^{T}$ is a vector of population parameters (fixed-effects), $\boldsymbol{a}_{i}=\left(a_{i 0}, a_{i 1}, a_{i 2}\right)^{T}$ is a vector of random-effects, and $\epsilon_{i j}$ is the measurement error with the ST distribution at time $t_{i j}$. In addition, in order to avoid too small or large estimates which may be unstable, we standardize the time-varying covariate CD4 cell counts (each CD4 value is subtracted by mean 375.46 and divided by standard deviation 228.57) and rescale the original time (in days) so that the time scale is between 0 and 1 .

Viral dynamic models can be formulated through a system of ordinary differential equations (Ding and Wu (1999), Huang et al. (2006)). Due to the limitations of current assays, only two infected cell compartments can be identified which are believed to produce a biphasic viral decay. Based on biological and clinical arguments, Ding and Wu (1999) proposed approximating a possibly multi-phase exponential decay model by the biphasic model

$$
y(t)=\ln \{V(t)\}+e(t)=\ln \left(e^{p_{1}-\lambda_{1} t}+e^{p_{2}-\lambda_{2} t}\right)+e(t),
$$

where $V(t)$ is the plasma HIV-1 RNA levels (viral load) at time $t, \lambda_{1}$ and $\lambda_{2}$ are called the first- and second-phase viral decay rates, which may represent the minimum turnover rate of productively infected cells and that of latently or longlived infected cells, respectively (Ding and Wu (1999)). The parameters $p_{1}$ and $p_{2}$ are macro-parameters; $e^{p_{1}}+e^{p_{2}}$ is the baseline viral load at time $t=0$. It is generally assumed that $\lambda_{1}>\lambda_{2}$, which assures that the model is identifiable and is appropriate for empirical studies (Ding and $\mathrm{Wu}(1999)$ ).

Because the inter-subject variations are substantial, one proposed NLME models for inference. At the first level, we specify a nonlinear model for individual trajectory based on the model (3.2) that depends on subject-specific parameters. At the second level, those parameters are modeled as a function of covariates with fixed-effects and random-effects. It was noted that the viral decay rates may vary over time because it depends on some phenomenologic parameters that hide considerable microscopic complexity and change over time. Negative values of the decay rates may correspond to viral increase and lead to viral rebound. It was suggested that variation in the dynamic parameters may be partially associated with CD4 cell count. When covariates change over time, the second-level model can be offered to describe the association between changes in parameters and changes in covariate values ( $\mathrm{Wu}(2002))$. Thus, we consider the 
following ST-NLME model for HIV dynamics.

$$
\begin{aligned}
& y_{i j}=\ln \left(e^{p_{i 1}-\lambda_{i 1} t_{i j}}+e^{p_{i 2}-\lambda_{i j 2} t_{i j}}\right)+e_{i j}, \\
& \beta_{i j 1}=p_{i 1}=\beta_{1}+b_{i 1}, \quad \beta_{i j 2}=\lambda_{i 1}=\beta_{2}+b_{i 2}, \\
& \beta_{i j 3}=p_{i 2}=\beta_{3}+b_{i 3}, \quad \beta_{i j 4}=\lambda_{i j 2}=\beta_{4}+\beta_{5} z_{i j}^{*}+b_{i 4},
\end{aligned}
$$

where $y_{i j}$ is the natural ln-transformation of the viral load for the $i$ th subject at time $t_{i j}\left(i=1,2, \ldots, n, j=1,2, \ldots, n_{i}\right)$ and $\boldsymbol{e}_{i}=\left(e_{i 1}, \ldots, e_{i n_{i}}\right)^{T}$ represents within-individual random error which follows the ST distribution, $z_{i j}^{*}$ is referred to as a summary of the true (but unobserved) CD4 value for the $i$ th subject at time $t_{i j}, \boldsymbol{\beta}_{i j}=\left(\beta_{i j 1}, \beta_{i j 2}, \beta_{i j 3}, \beta_{i j 4}\right)^{T}$ and $\boldsymbol{\beta}=\left(\beta_{1}, \beta_{2}, \ldots, \beta_{5}\right)^{T}$ are individual parameters for the $i$ th subject at time $t_{i j}$ and population parameters, respectively, $\boldsymbol{b}_{i}=\left(b_{i 1}, \ldots, b_{i 4}\right)^{T}$ is individual random-effects. Note that the ln-transformation of the viral load is taken here to stabilize the variation of measurement errors and to speed up estimation algorithm. The model (3.3) is a natural extension of the model (3.2) to accommodate time-varying covariate CD4 into the secondphase decay rate for capturing viral rebound. It is noted that the joint model conducted by Huang and Dagne (2011) is a special case of the proposed models with an SN distribution (3.1) and (3.3) when they are tailored as follows: (i) the censored values are imputed by half of LOD in such a way that a fixed value is chosen to represent all observations below LOD rather than using a fully Bayesian predictive distribution to impute the observations below LOD; (ii) the unknown nonparametric smooth function for the covariate process is substituted by a known quadratic polynomial; and (iii) an SN distribution, which is a special case of an ST distribution when degrees of freedom are large, is assumed for model errors.

\subsection{Modeling implementation}

In this section, we analyze the AIDS data set described in Section 1 to illustrate our method using the ST-NLME model (3.3) in conjunction with the ST covariate model (3.1), and the corresponding specifications of prior distributions. As shown in Fig. 1 (top panel), the histograms of viral load in ln scale and CD4 cell count clearly indicate their asymmetric nature, and it is appropriate to fit an ST joint model to the data set. With this consideration, the following two statistical models with different distributions of random errors for both the response model (3.3) and the covariate model (3.1) are employed to compare their performance.

- Model I: A model with independent multivariate $t$-distributions of random errors for both the covariate model and the response model;

- Model II: A model with independent multivariate ST distributions of random errors for both the covariate model and the response model.

We investigate the following three scenarios. First, since a (symmetric) $t$ distribution is a special case of an ST distribution when skewness parameter is zero, we investigate how an (asymmetric) ST distribution for model error contributes to modeling results and parameter estimation in comparison with 
a (symmetric) $t$-distribution. Second, we estimate the model parameters by using the 'naive' method (denoted by NV), which ignores measurement errors in CD4. That is, the 'naive' method only uses the observed CD4 values $z_{i j}$ rather than true (unobservable) CD4 values $z_{i j}^{*}$ in the response model (3.3) in which case the joint NLME model (2.5) reverts to a regular NLME model. We use it as a comparison to the joint modeling (JM) approach proposed in Section 2. This comparison attempts to investigate how the measurement errors in CD4 contribute to modeling results. Finally, when covariates are measured with errors, a common approach is the so-called two-step (TS) method (Higgins et al. (1997)): the first step estimates the 'true' covariate values based on the covariate model (3.1); at the second step the covariate in the response model (2.5) is substituted by the estimate from the first step. Thus, the two-step (TS) method is provided to compare the performance with the JM method.

The progress in Bayesian posterior computation due to MCMC procedures has made it possible to fit increasingly complex statistical models (Huang et al. (2006), Lunn et al. (2000)) and entailed the wish to determine the best fitting model in a class of candidates. Thus, it has become more and more important to develop efficient model selection criteria. A recent publication by Spiegelhalter et al. (2002) suggested a generalization of AIC and related also to BIC that is deviance information criterion (DIC). Since the structure of DIC allows for automatic computation in WinBUGS software (Lunn et al. (2000)), we will use DIC to compare the models in this paper although other criteria such as AIC and BIC can be chosen. As with other model selection criteria, we caution that DIC is not intended for identification of the 'correct' model, but rather merely as a method of comparing a collection of alternative formulations. In our models with different distribution specifications for model errors, DIC can be used to find out how assumption of an ST distribution contributes to virologic response in comparison with that of a $t$-distribution and how the proposed joint modeling approach influences parameter estimation compared with the 'naive' method and two-step method.

To carry out the Bayesian inference, we need to specify the values of the hyper-parameters in the prior distributions. In the Bayesian approach, we only need to specify the priors at the population level. We take weakly informative prior distribution for the parameters in the models. In particular, (i) fixed-effects were taken to be independent normal distribution $N(0,100)$ for each component of the population parameter vectors $\boldsymbol{\alpha}$ and $\boldsymbol{\beta}$. (ii) For the precision parameters $\tau^{2}$ and $\sigma^{2}$ we assume a limiting non-informative inverse gamma prior distribution, $I G(0.01,0.01)$ so that the distribution has mean 1 and variance 100 . (iii) The priors for the variance-covariance matrices of the random-effects $\boldsymbol{\Sigma}_{a}$ and $\boldsymbol{\Sigma}_{b}$ are taken to be inverse Wishart distributions $I W\left(\boldsymbol{\Omega}_{1}, \rho_{1}\right)$ and $I W\left(\boldsymbol{\Omega}_{2}, \rho_{2}\right)$ with covariance matrices $\boldsymbol{\Omega}_{1}=\operatorname{diag}(0.01,0.01,0.01), \boldsymbol{\Omega}_{2}=\operatorname{diag}(0.01,0.01,0.01,0.01)$ and degree of freedom $\rho_{1}=\rho_{2}=5$, respectively. (iv) The degree of freedom parameters $\nu_{\epsilon}$ and $\nu_{e}$ follow truncated exponential distribution with $\nu_{10}=\nu_{20}=0.5$. (v) For each of the skewness parameters $\delta_{e}$ and $\delta_{\epsilon}$, we choose independent nor- 
mal distribution $N(0,100)$, where we assume that $\boldsymbol{\delta}_{e_{i}}=\delta_{e} \mathbf{1}_{n_{i}}$ and $\boldsymbol{\delta}_{\epsilon_{i}}=\delta_{\epsilon} \mathbf{1}_{n_{i}}$ to indicate that we are interested in skewness of overall viral load data and overall CD4 cell count data. The MCMC sampler was implemented using WinBUGS software and the program codes are available from authors on request. We chose the initial values from the previous studies ( $\mathrm{Wu}$ and Zhang (2002), Liu and Wu (2007), Huang and Dagne (2011)) and among others. When the MCMC implementation is applied to the actual clinical data, convergence of the generated samples is assessed using standard tools (such as trace plots), which are the same as those used by Huang et al. (2006). That is, we graphically monitor the MCMC samples for parameters within WinBUGS and see if consecutive samples move randomly towards a stationary directions, indicating that MCMC sampler is not "sticky"; in addition, an informal check of convergence is also conducted based on graphical techniques (Lunn et al. (2000)). See Fig. 1 in publication by Huang et al. (2006) for details. Along with the convergence diagnostics, one long chain was run which may be more efficient with the following considerations. We propose that, after an initial number of 50,000 burn-in iterations, every 40th MCMC sample is retained from the next 400,000. Thus we obtain 10,000 samples of targeted posterior distributions of the unknown parameters for statistical inference.

\subsection{Comparison of joint modeling results}

The Bayesian joint modeling approach in conjunction with the NLME response model (3.3) and the covariate measurement error model (3.1) with the $t$ or ST distribution for the random errors was used to fit the viral load data with left-censoring and CD4 data with measurement error. In this section, we will report analysis results of the three scenarios proposed in Subsection 3.2.

The population posterior mean (PM), the corresponding standard deviation (SD) and 95\% credible interval for fixed-effects parameters based on the three methods (JM, NV and TS methods) are presented in Table 1. The following findings are observed for estimated results of parameters based on JM method. (i) In the response model (3.3), for the most interested parameters $\left(\beta_{2}, \beta_{4}, \beta_{5}\right)$ in this research, the estimates of $\beta_{2}$ and $\beta_{4}$ based on Model I are smaller than those based on Model II, while the estimate of $\beta_{5}$ based on Model I which is coefficient of true CD4 covariate is larger than that based on Model II. All of them are significantly estimated for both Models since the $95 \%$ credible intervals do not contain zero. The results indicate that estimated parameters may be substantially biased if model distribution ignores skewness. For the precision parameter $\sigma^{2}$, the estimated value (0.90) based on Model I is much larger than that (0.01) based on Model II. (ii) For parameter estimates of the CD4 covariate model (3.1), the estimates of intercept $\alpha_{0}$ and coefficient $\alpha_{2}$ based on Model I are larger than those based on Model II; however, the estimate of the coefficient $\alpha_{1}$ are almost identical for the both models. For the precision parameter $\tau^{2}$, the estimated value (0.13) based on the Model I is larger than that (0.09) based on the Model II.

Figure 2 displays the three randomly selected individual estimates of viral 


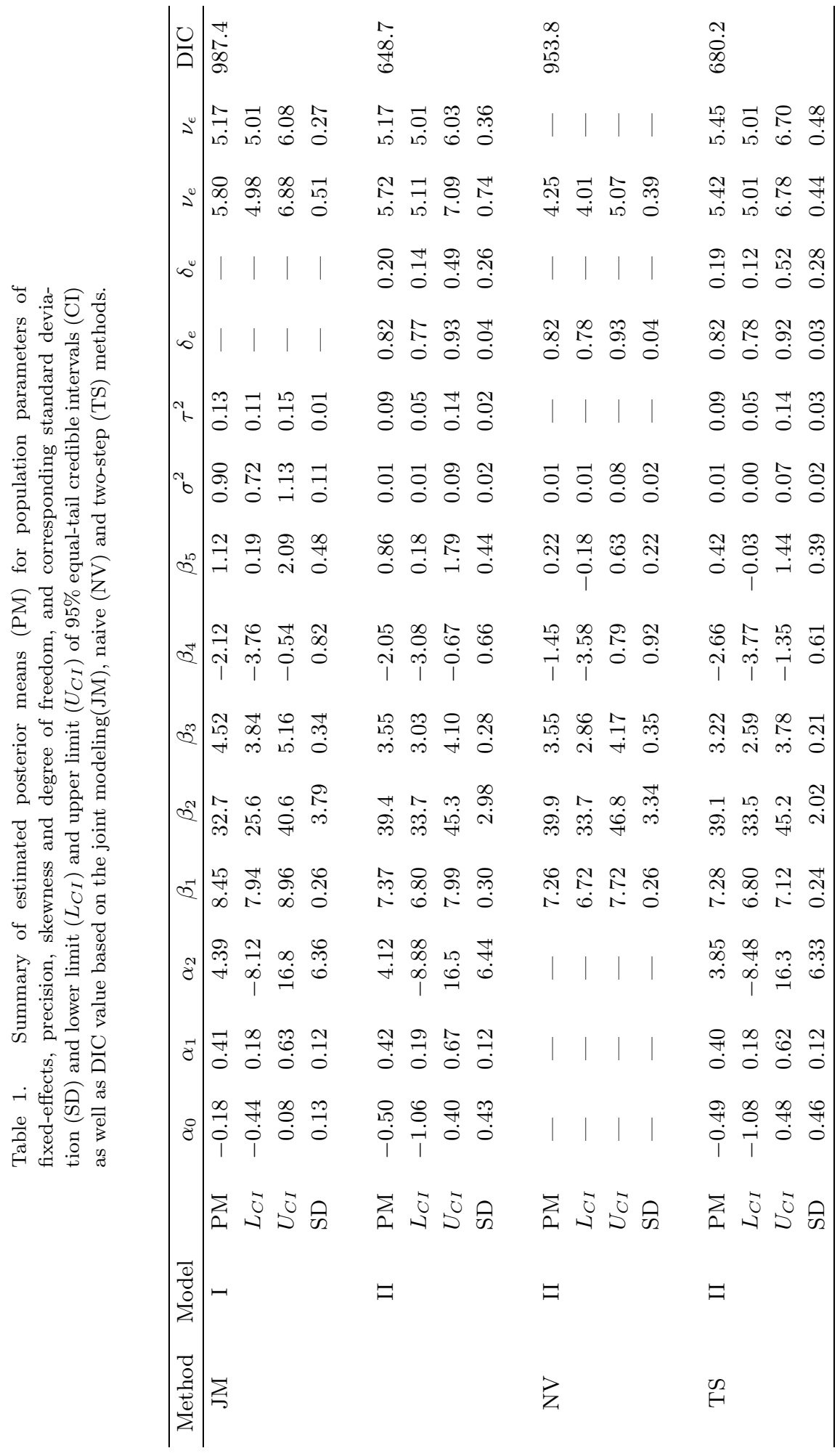



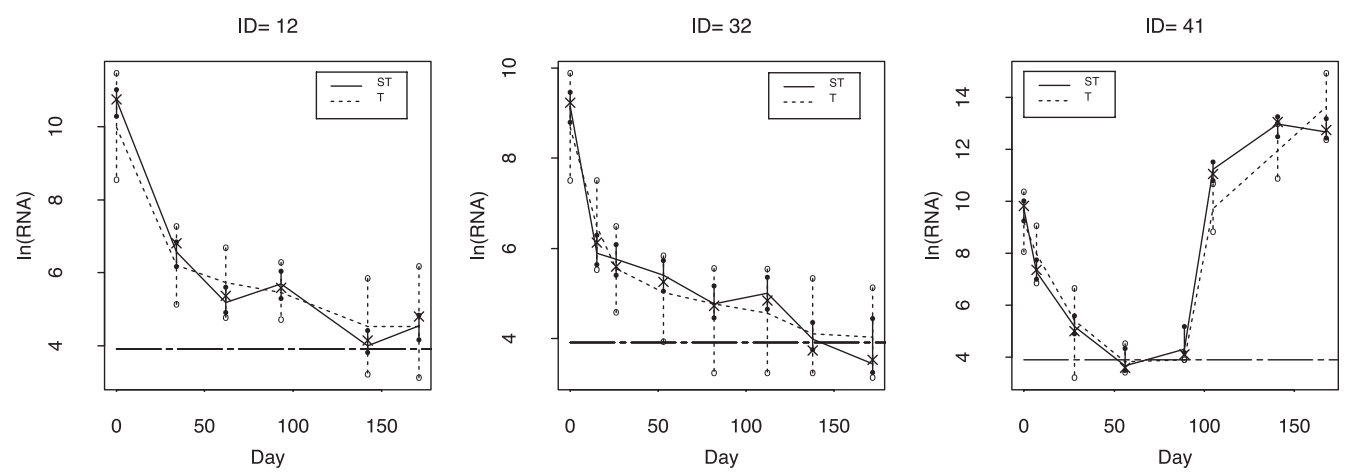

Figure 2. The individual fitted curves of viral load for three randomly selected patients based on the joint models with the $t$-distribution, denoted by $\mathrm{T}$ (dotted line) or ST distribution (solid line) for model error. The respective vertical dotted line (T) ended with ' $\circ$ ' and solid line (ST) ended with ' $\bullet$ ' on each fitted value are the $95 \%$ credible interval (CI) associated with the fitted value. The observed values are indicated by sign crosses $(x)$. The horizontal line is below the detectable limit of viral load, $3.91=\ln (50)$.

load trajectories along with the associated $95 \%$ confidence interval on each fitted value obtained by using the JM method based on Models I (T) and II (ST). The following findings are observed from joint modeling results. (i) The estimated individual trajectories for Model II where the random error is assumed to have ST distribution fit the originally observed values more closely than those for Model I where the random error is assumed to have $t$-distribution. Note that the lack of smoothness in Model II estimates of individual trajectories is understandable since a random component $\boldsymbol{w}_{e_{i}}$ was incorporated in the expected function (see (2.5) for details) according to the stochastic representation feature of the ST distribution for "chasing the data" to this extent. (ii) Overall, the $95 \%$ credible interval (CI) associated with predicted value from Model I is wider than the corresponding 95\% CI from Model II. (iii) All the 95\% CIs from Model II cover the true (observed) viral load values, while some of $95 \%$ CIs from Model I do not; for example, for patient 41, the observed value at day 105 is 11.05 , but the corresponding $95 \%$ CI from Model I is $(8.83,10.66)$ with the fitted value 9.73 . (iv) Model II provides better prediction to the observed values below LOD than Model I does such as the last measurement which is below LOD for the patient 32 .

From the model fitting results, we have seen that, in general, both the models provided a reasonably good fit to the observed data for most patients in our study, although the fitting for a few patients was not completely satisfactory due to unusual viral load fluctuation patterns for these patients, particularly for Model I. To assess the goodness-of-fit of the proposed models, the diagnosis plots for the two models, the observed values versus the fitted values (left panel) and ST or student $t$ (T) Q-Q plots (right panel), are presented in Fig. 3. It was seen from the plots of the observed values versus the fitted values (left panel) that Model II provided better fit to observed data, compared with Model I. 

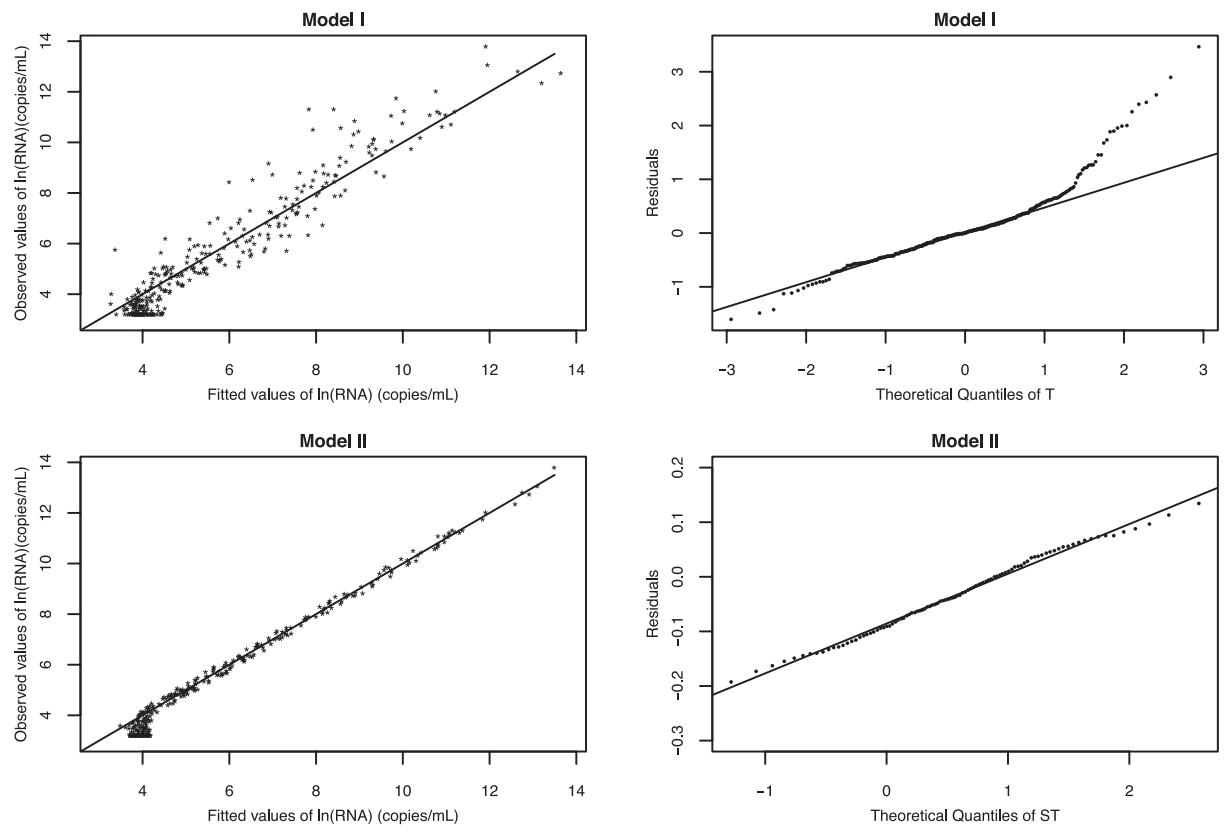

Figure 3. Goodness-of-fit: Observed values versus fitted values of $\ln (\mathrm{RNA})$ (left panel; ST and student $t$ (T) Q-Q plots with line (right panel).

This result can be also explained by examining the ST and T Q-Q plots of the residuals (right panel) that both plots show the existence of outliers, but it is clearly indicated that Model II only has few outliers and thus fits observed data better than Model I.

In order to further investigate whether Model II can provide better fit to the data than Model I, we calculate estimated DICs for both models based on the JM method and find that the DIC value (987.4) for Model I (with student $t$ random error) is larger than that (648.7) for Model II (with ST random error). As mentioned before, it is hard to tell which model is 'correct' but which one fits data better. Furthermore, the model which fits data better may be more accurate to describe mechanism of HIV infection and CD4 changing process, thus needs more attention for patient treatment. Therefore, based on the DIC criterion, the results indicate that Model II is the better fitting model, supporting the contention of a departure from normality. These results are consistent with those in diagnosis of the goodness-of-fit displayed in Fig. 3 indicating that Model II outperforms Model I. In summary, our results may suggest that it is very important to assume an ST distribution for the response model and the CD4 covariate model in order to achieve reliable results, in particular if the data exhibit skewness. Along with these observations, we will further report our results in detail only for Model II in Subsection 3.4 below. 


\subsection{Estimation results based on Model II}

For comparison, we used the 'naive' (NV) method to estimate the model parameters presented in Table 1 where the raw (observed) CD4 values $z_{i j}$, rather than the true (unobserved) CD4 values $z_{i j}^{*}$ associated with the second-phase viral decay rate $\lambda_{i j 2}$, are substituted in the response model (3.3). It can be seen that there are important differences in the estimates for the parameters $\beta_{4}$ and $\beta_{5}$ which are directly associated with whether or not ignoring potential CD4 measurement errors for inference. The NV method may produce biased estimates and may substantially underestimate the covariate $\mathrm{CD} 4$ effect $\left(\beta_{5}\right)$. The estimated $\mathrm{SD}$ for the CD4 effect $\left(\beta_{5}\right)$ using the JM method is twice larger than that using the naive method, probably because the JM method incorporates the variation from fitting the CD4 process. The difference of the NV estimates and the JM estimates, due to whether or not ignoring potential CD4 measurement errors for inference indicates that CD4 measurement errors can not be ignored in the analysis. The results suggest that estimated parameters may be substantially biased if measurement errors in CD4 covariate are ignored. We also obtain estimated DIC values of 953.8 based on the NV method. We can see from the estimated DIC values that the JM approach provides a better fit to the data in comparison with the NV method. Thus, it is important to take the measurement errors into account when covariates are measured with errors.

Table 1 also presents estimated population parameters using the two-step (TS) method. We can see the TS estimates and the JM estimates are somewhat different; in particular, there are important differences in the estimates for the parameters $\beta_{4}$ and $\beta_{5}$, which are directly associated CD4 covariate. For the parameter $\boldsymbol{\beta}$ of main interest, the JM method appears to give larger standard deviations (SD) than the TS method, probably because it incorporates the variation from fitting the CD4 process, whereas the TS method fails to take CD4 uncertainty into account. So the standard deviations produced by the TS method may be underestimated.

The estimated results presented in Table 1 based on the JM method indicate that the estimates of the parameters in the CD4 covariate model (3.1) suggest a significant overall increase in CD4 after the treatment, and both individual variation and CD4 measurement error appear substantial. The estimates for the individual-specific first-phase viral decay rate $\hat{\lambda}_{1 i}=\hat{\beta}_{2}+b_{i 2}$ in the viral dynamic model (3.3), ranged from 37.16 to 41.52 with standard deviation 1.28 , indicate that the overall first-phase viral decay is significant with a substantial variation across subjects; the estimate of population parameter $\beta_{2}$ (SD) is 39.4 (2.98), and the estimate of variance of random-effects $b_{i 2}$ is 4.78 . The estimates of the individual-specific second-phase viral decay rate (i.e., $\hat{\lambda}_{i j 2}=-2.05+0.86 z_{i j}^{*}+b_{i 4}$ with the estimated variance of random-effects $b_{i 4}$ being 9.71) appears positively and significantly associated with the true (unobservable) CD4 value over time. This is consistent with the findings reported by Wu (2002) and Wu et al. (2008). The true CD4 process has a significantly positive effect on the second-phase viral decay rate; this finding confirms that the $\mathrm{CD} 4$ covariate may be a significant 
(a): Mean $=39.03, \mathrm{SD}=1.28, \mathrm{CV}=5.29 \%$

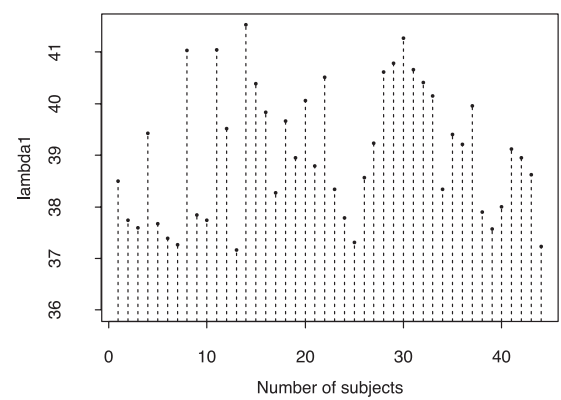

(b): $r=-0.337, p=0.0272$

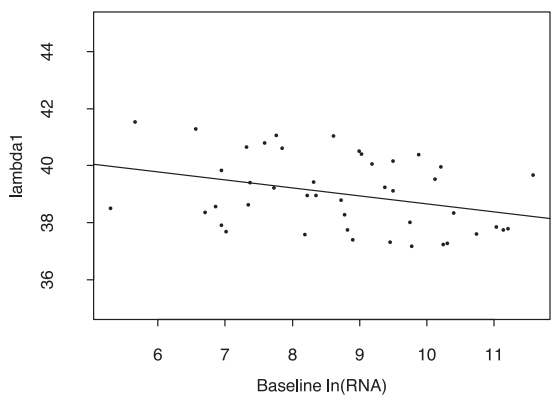

Figure 4. (a): The individual estimates of the first-phase decay rate $\lambda_{1}$. (b): The correlation between baseline $\ln (\mathrm{RNA})$ levels and the individual estimates of the first-phase decay rate $\lambda_{1}$. The solid line is robust (MM-estimator) linear regression fit. The correlation coefficients $(r)$ and $p$-values were obtained from Spearman rank correlation test.

predictor on the second-phase viral decay rate during the process. More rapid increase in CD4 cell count may be associated with faster viral decay in the late stage. This may be explained by the fact that higher CD4 cell count suggest a higher turnover rate of lymphocyte cells, which may cause a positive correlation between viral decay and the CD4 cell count.

Recent research findings indicate that the decay rates of viral responses to a treatment is a potentially useful marker for antiretroviral potency (Ding and Wu (1999)). Individual-specific parameter estimates are very important and have implications for the tailoring of treatment for individual patients with AIDS. As mentioned previously, in the response model $(3.3)$, the intercepts $\left(p_{i 1}, p_{i 2}\right)$ are macro-parameters which have no interpretable biological meanings, whereas the first- and second-phase viral decay rates $\lambda_{i 1}$ and $\lambda_{i j 2}$ represent the minimum turnover rate of productively infected cells and that of latently or long-lived infected cells, respectively. Note that $\lambda_{i 1}$ is dependent of subjects only, while $\lambda_{i j 2}$ is considered to be dependent of both subjects and time points. Thus as an example, we present the individual-specific parameter estimates of $\lambda_{i 1}$ based on the JM method in Fig. 4(a). The results suggest a significant between-subject variation observed since the coefficient of variation (CV) is $5.29 \%$.

We further investigate the relation between the estimated individual viral decay rates $\lambda_{i 1}$ and baseline $\ln$ (RNA) levels, which is shown in Fig. 4(b). The subject-specific estimates of $\lambda_{i 1}$ show significantly negative correlation $(r=$ -0.337 and $p=0.0272$ ) with baseline $\ln (\mathrm{RNA})$ levels. This can be explained by the fact that the slower viral decay rate may result in a higher viral load. The negative correlation result here is also consistent with the findings obtained by $\mathrm{Wu}$ et al. (2003, 2004).

The results also indicate that the estimated population first- and secondphase rates of change in viral load $\hat{\lambda}_{1}=39.4$ and $\hat{\lambda}_{2}(t)=-2.05+0.86 z^{*}(t)$, respectively, where $z^{*}(t)$ is the standardized true CD4 value at time $t$ which may be interpreted as the 'regularized' covariate value. Thus, the population viral load 
process may be approximated by $\hat{V}(t)=\exp \left[7.37-\hat{\lambda}_{1} t\right]+\exp \left[3.55-\hat{\lambda}_{2}(t) t\right]$. Since the second-phase viral decay rate $\left(\lambda_{2}(t)\right)$ is significantly associated with the true CD4 values (due to statistically significant estimate of $\beta_{5}$ ), this suggests that the viral load change $V(t)$ may be significantly associated with the true CD4 process. Note that, although the true association described above may be complicated, the simple approximation considered here may provide a rough guidance and point to further research. In addition, the estimate of within-subject precision parameter $\sigma^{2}$ in Model II (0.01) is much smaller than that in Model I (0.90), which indicates that gain in significant efficiency for Model II relative to Model I is observed for the precision estimation. This is expected because high variability and the skewness are interrelated to a certain extent.

The estimated results based on the JM method in Table 1 show that the skewness parameters in viral load (0.82) and CD4 cell count (0.20) with degrees of freedom being 5.80 and 5.17, respectively, are significantly positive, which confirm the heavy right tail skewness of the viral load and fairly right tail of the CD4 cell count. Thus, it may suggest that accounting for significant skewness, when the data exhibit skewness, provides a better model fit to the data and gives more accurate estimates to the parameters.

\section{Concluding discussion}

This paper developed a general joint modeling approach for Bayesian nonlinear mixed-effects models with an ST distribution for longitudinal data with skewness characteristics, left-censoring due to LOD and measurement errors in covariates that may be preferred over those with a standard $t$-distribution. The results also indicate that the JM method outperformed the NV and TS methods in the sense that it produces more robust and less biased parameter estimates. The proposed JM method is quite general, and so can be used to other applications. This kind of ST modeling approach is important in many biostatistical application areas, allowing accurate inference of parameters while adjusting for the data with skewness. The ST distribution is shown to provide an alternative to (symmetric) $t$-distribution that is often assumed in statistical models.

As discussed in Subsection 2.2, the ST model (2.5) can be reverted to the SN model, the standard student $t(\mathrm{~T})$ model and the standard normal model. We investigated the SN and normal models in the same procedure as we have done in this paper. The following findings are observed. (i) The SN and normal models provide similar results in comparison with the $\mathrm{ST}$ and $\mathrm{T}$ models, respectively, in terms of estimated parameters, but their estimates are somewhat different for some parameters. For example, for the JM method the estimate (0.82) of the skewness parameter $\delta_{e}$ based on the model with the ST distribution (Model II) is less than that (1.43) based on the model with the SN distribution. This may be due to the fact that an additional parameter, the degree of freedom $\left(\nu_{e}\right)$ for heaviness in the tails is estimated to have 5.72 trading-off the effect of skewness. (ii) For comparing model fitting, we also obtain DIC values of 717.4 and 1024.3 for the SN and normal models, which are larger than those for the ST and T 
models, respectively. It indicates that the ST and T models perform better than the SN and normal models, respectively. The detailed results based on the SN and the normal models are omitted in this paper to save space.

We have assumed that there is an underlying nonparametric smooth process that captures the main features of the covariate (CD4) trajectories, and we have used a flexible ST nonparametric mixed-effects model to approximate this process using a regression spline method. Note that the approximation is only an empirical one and may not be true but unknown CD4 path. Thus, the fitted CD4 values may not be the 'true' CD4 values but rather 'regularized' CD4 values which help alleviating measurement errors in observed CD4 cell counts. We have assumed that the viral dynamic model continues to hold for non-detectable (censored) viral loads. This assumption may be reasonable since the dynamic model considered here is a natural extension of a biologically justified model. The validity of the model for non-detectable viral loads, however, cannot be verified based on the observed data since the non-detectable values are not observed so there is some uncertainty associated with these values. It is possible that, when viral loads are below detection limit, the viral elimination and production mechanism could be different. In such case, a sensitivity analysis under various plausible models for non-detectable viral loads may be helpful. Note that any assumed model for unobserved (non-detectable) viral loads cannot be rejected by statistical tests based on the observed data. At the absence of other theoretical justification, the assumption in this article appears to be reasonable.

The results indicate that with skew distribution assumption, there is potential to gain efficiency and accuracy in estimating certain parameters when the normality assumption does not hold in the data. The models considered in this paper can be easily fitted using MCMC procedure. Moreover, the proposed modeling approach for the ST-NLME joint model is very easy to implement using the WinBUGS package that is available publicly and has a computational cost similar to the normal version of the model due to the features of its hierarchically stochastic representations. This makes our approach quite powerful and accessible to practicing statisticians in the fields. It is noted that Huang and Dagne (2011) investigated the NLME model with assuming both the model error and random-effects to have skew distributions and found that the modeling results based on the skew distribution for random-effects provided a similar performance to those based on a normal distribution for random-effects. Thus the random-effects were assumed to be normal here although more robust specification of distributions can be employed. It is our purpose that this paper is to demonstrate the proposed models and methods with various scenarios for real data analysis in comparison of symmetric distributions with asymmetric distributions for model errors, although a limited simulation study may be conducted to confirm our results from different model specifications and the corresponding methods. However, since this paper investigated many different scenarios-based models and methods with real data analysis, the complex natures considered in this article, especially, skew distributions involved, will pose some challenges 
for such simulation study which requires additional efforts, but beyond the purpose of this article. We believe that a further study to investigate these related problems may be warranted and we could report these interesting results in the near future.

Appendix: Multivariate skew- $t$ distributions

Different versions of the multivariate skew- $t$ (ST) distributions have been proposed and used in the literature (Azzalini and Capitanio (2003), Branco and Dey (2001), Jara et al. (2008), Sahu et al. (2003) and among others). For completeness, this section is started by briefly summarizing the multivariate ST distribution that will be used in defining the ST joint models considered in this paper. For detailed discussions on properties and differences among various versions of ST distributions, see the references above. We consider a multivariate ST distribution introduced by Sahu et al. (2003) which is suitable for a Bayesian inference since it is built using conditional method and is defined below.

An $m$-dimensional random vector $\boldsymbol{Y}$ follows an $m$-variate ST distribution if its probability density function (pdf) is given by

$$
f(\boldsymbol{y} \mid \boldsymbol{\mu}, \boldsymbol{\Sigma}, \boldsymbol{\Delta}(\boldsymbol{\delta}), \nu)=2^{m} t_{m, \nu}(\boldsymbol{y} \mid \boldsymbol{\mu}, \boldsymbol{A}) P(\boldsymbol{V}>\mathbf{0}),
$$

where $\boldsymbol{A}=\boldsymbol{\Sigma}+\boldsymbol{\Delta}^{2}(\boldsymbol{\delta})$, we denote the $m$-variate $t$ distribution with parameters $\boldsymbol{\mu}, \boldsymbol{A}$ and degrees of freedom $\nu$ by $t_{m, \nu}(\boldsymbol{\mu}, \boldsymbol{A})$ and the corresponding pdf by $t_{m, \nu}(\boldsymbol{y} \mid \boldsymbol{\mu}, \boldsymbol{A})$ henceforth, $\boldsymbol{V}$ follows the $t$ distribution $t_{m, \nu+m}$. We denote this distribution by $S T_{m, \nu}(\boldsymbol{\mu}, \boldsymbol{\Sigma}, \boldsymbol{\Delta}(\boldsymbol{\delta}))$. In particular, when $\boldsymbol{\Sigma}=\sigma^{2} \boldsymbol{I}_{m}$ and $\boldsymbol{\Delta}(\boldsymbol{\delta})=$ $\delta \boldsymbol{I}_{m}$, the equation (A.1) simplifies to

$$
\begin{aligned}
f(\boldsymbol{y} \mid \boldsymbol{\mu}, & \left.\sigma^{2}, \delta, \nu\right) \\
= & 2^{m}\left(\sigma^{2}+\delta^{2}\right)^{-m / 2} \frac{\Gamma((\nu+m) / 2)}{\Gamma(\nu / 2)(\nu \pi)^{m / 2}}\left\{1+\frac{(\boldsymbol{y}-\boldsymbol{\mu})^{T}(\boldsymbol{y}-\boldsymbol{\mu})}{\nu\left(\sigma^{2}+\delta^{2}\right)}\right\}^{-(\nu+m) / 2} \\
& \times T_{m, \nu+m}\left[\left\{\frac{\nu+\left(\sigma^{2}+\delta^{2}\right)^{-1}(\boldsymbol{y}-\boldsymbol{\mu})^{T}(\boldsymbol{y}-\boldsymbol{\mu})}{\nu+m}\right\}^{-1 / 2} \frac{\delta(\boldsymbol{y}-\boldsymbol{\mu})}{\sigma \sqrt{\sigma^{2}+\delta^{2}}}\right],
\end{aligned}
$$

where $T_{m, \nu+m}(\cdot)$ denotes the cumulative distribution function (cdf) of $t_{m, \nu+m}\left(\mathbf{0}, \boldsymbol{I}_{m}\right)$. However, unlike in the SN distribution (Sahu et al. (2003)), the ST density can not be written as the product of univariate ST densities. Here $\boldsymbol{Y}$ are dependent but uncorrelated.

According to Lemma 1 of Azzalini and Capitanio (2003), if $\boldsymbol{Y}$ follows $S T_{m, \nu}(\boldsymbol{\mu}, \boldsymbol{\Sigma}, \boldsymbol{\Delta}(\boldsymbol{\delta}))$, it can be represented by

$$
\boldsymbol{Y}=\boldsymbol{\mu}+u^{-1 / 2} \boldsymbol{X}
$$

where $u$ follows a Gamma distribution $\Gamma(\nu / 2, \nu / 2)$ which is independent of $\boldsymbol{X}$ and $\boldsymbol{X}$ follows an $m$-dimensional skew-normal (SN) distribution, denoted by $S N_{m}(\mathbf{0}, \boldsymbol{\Sigma}, \boldsymbol{\Delta}(\boldsymbol{\delta}))$. It follows from (A.3) that $\boldsymbol{Y} \mid u \sim S N_{m}(\boldsymbol{\mu}, \boldsymbol{\Sigma} / u, \boldsymbol{\Delta}(\boldsymbol{\delta}))$. By 
Proposition 1 of Arellano-Valle et al. (2007), the SN distribution of $\boldsymbol{Y}$ conditional on $u$ has a convenient stochastic representation as follows.

$$
\boldsymbol{Y}=\boldsymbol{\mu}+\boldsymbol{\Delta}(\boldsymbol{\delta})\left|X_{0}\right|+u^{-1 / 2} \boldsymbol{\Sigma}^{1 / 2} X_{1},
$$

where $X_{0}$ and $X_{1}$ are two independent $N_{m}\left(\mathbf{0}, \boldsymbol{I}_{m}\right)$ random vectors. Note that the expression (A.4) provides a convenience device for random number generation and for implementation purpose. Let $\boldsymbol{w}=\left|X_{0}\right|$; then $\boldsymbol{w}$ follows an $m$-dimensional standard normal distribution $N_{m}\left(\mathbf{0}, \boldsymbol{I}_{m}\right)$ truncated in the space $\boldsymbol{w}>\mathbf{0}$ (i.e., the standard half-normal distribution). Thus, following Sahu et al. (2003), a hierarchical representation of (A.4) is given by

$$
\begin{aligned}
& \boldsymbol{Y} \mid \boldsymbol{w}, u \sim N_{m}(\boldsymbol{\mu}+\boldsymbol{\Delta}(\boldsymbol{\delta}) \boldsymbol{w}, \boldsymbol{\Sigma} / u), \quad \boldsymbol{w} \sim N_{m}\left(\mathbf{0}, \boldsymbol{I}_{m}\right) \boldsymbol{I}(\boldsymbol{w}>\mathbf{0}), \\
& u \sim \Gamma(\nu / 2, \nu / 2),
\end{aligned}
$$

Note that the ST distribution presented in (A.5) can be reduced to the following three special cases: (i) as $\nu \rightarrow \infty$ and $u \rightarrow 1$ with probability 1 (i.e., the last distributional specification is omitted), then the hierarchical expression (A.5) becomes an SN distribution $S N_{m}(\boldsymbol{\mu}, \boldsymbol{\Sigma}, \boldsymbol{\Delta}(\boldsymbol{\delta}))$; (ii) as $\boldsymbol{\Delta}(\boldsymbol{\delta})=\mathbf{0}$, then the hierarchical expression (A.5) is a standard multivariate $t$-distribution; (iii) as $\nu \rightarrow \infty, u \rightarrow 1$ with probability 1 , and $\boldsymbol{\Delta}(\boldsymbol{\delta})=\mathbf{0}$, then the hierarchical expression (A.5) reverts to a standard multivariate normal distribution.

\section{Acknowledgements}

The author is extremely grateful to the Editor and two anonymous reviewers for their insightful comments and suggestions that led to an improvement of the article. This research was partially supported by NIAID/NIH grant R03 AI080338 and MSP/NSA grant H98230-09-1-0053 to Huang, and NIMH grant R01MH040859-22 to Dagne.

\section{REFERENCES}

Acosta, E. P., Wu, H., Hammer, S. M., Yu, S., Kuritzkes, D. R., Walawander, A., Eron, J. J., Fichtenbaum, C. J., Pettinelli, C., Neath, D., Ferguson, E., Saan, A. J. and Gerber, J. G. for the Adult ACTG 5055 Protocol Team (2004). Comparison of two indinavir/ritonavir regimens in treatment of HIV-infected individuals, JAIDS, 37, 1358-1366.

Arellano-Valle, R. B., Bolfarine, H. and Lachos, V. H. (2007). Bayesian inference for skewnormal linear mixed models, Journal of Applied Statistics 34, 663-682.

Azzalini, A. and Capitanio, A. (2003). Distributions generated by perturbation of symmetry with emphasis on a multivariate skew $t$ distributions, J. R. Stat. Soc. Ser. B, 65, 367-389.

Branco, M. D. and Dey, D. K. (2001). A general class of multivariate skew-elliptical distributions, J. Multivar. Anal., 79, 99-113.

Carroll, R. J., Ruppert, D., Stefanski, L. A. and Crainiceanu, C. M. (2006). Measurement Error in Nonlinear Models: A Modern Perspective, 2nd Ed., Chapman \& Hall, London.

Davidian, M. and Gilitinan, D. M. (1995). Nonlinear Models for Repeated Measurement Data, Chapman \& Hall, London.

Ding, A. A. and Wu, H. (1999). Relationships between antiviral treatment effects and biphasic viral decay rates in modeling HIV dynamics, Mathematical Biosciences, 160, 63-82. 
Higgins, M., Davidian, M. and Gilitinan D. M. (1997). A two-step approach to measurement error in time-dependent covariates in nonlinear mixed-effects models, with application to IGF-I pharmacokinetics, J. Am. Stat. Assoc., 92, 436-448.

Huang, Y. and Dagne G. (2011). A Bayesian approach to joint mixed-effects models with a skew-normal distribution and measurement errors in covariates, Biometrics, 67, 260-269.

Huang, Y., Liu, D. and Wu, H. (2006). Hierarchical Bayesian methods for estimation of parameters in a longitudinal HIV dynamic system, Biometrics, 62, 413-423.

Hughes, J. P. (1999). Mixed effects models with censored data with applications to HIV RNA levels, Biometrics, 55, 625-629.

Jara, A., Quintana, F. and Martin, E. S. (2008). Linear mixed models with skew-elliptical distributions: A Bayesian approach, Comput. Stat. Data Anal., 52, 5033-5045.

Liu, W. and Wu, L. (2007). Simultaneous inference for semiparametric nonlinear mixed-effects models with covariate measurement errors and missing responses, Biometrics, 63, 342-350.

Lunn, D. J., Thomas, A., Best, N. and Spiegelhalter, D. (2000). WinBUGS - a Bayesian modelling framework: Concepts, structure, an extensibility, Stat. Comput., 10, 325-337.

Rubin, D. B. (1976). Inference and missing data, Biometrika, 63, 581-592.

Sahu, S. K., Dey, D. K. and Branco, M. D. (2003). A new class of multivariate skew distributions with applications to Bayesian regression models, The Canadian Journal of Statistics, 31, $129-150$.

Spiegelhalter, D. J., Best, N. G., Carlin, B. P. and Van der Linde, A. (2002). Bayesian measures of model complexity and fit (with Discussion), J. R. Stat. Soc. Ser. B, 64, 583-639.

Verbeke, G. and Lesaffre, E. (1996). A linear mixed-effects model with heterogeneity in randomeffects population, J. Am. Stat. Assoc., 91, 217-221.

Wu, L. (2002). A joint model for nonlinear mixed-effects models with censoring and covariates measured with error, with application to AIDS studies, J. Am. Stat. Assoc., 97, 955-964.

$\mathrm{Wu}, \mathrm{L} ., \mathrm{Hu}, \mathrm{X}$. J. and Wu, H. (2008). Joint inference for nonlinear mixed-effects models and time-to-event at the presence of missing data, Biostatistics, 9, 308-320.

$\mathrm{Wu}, \mathrm{H}$. and Zhang, J. T. (2002). The study of long-term HIV dynamics using semi-parametric non-linear mixed-effects models, Stat. Med., 21, 3655-3675.

Wu, H., Mellors, J., Ruan, P. et al. (2003). Viral Dynamics and their relations to baseline factors and logn-term virologic responses in treatment-naive HIV-1 infected patients receiving abacavir in combination with HIV-1 protease inhibitors, JAIDS, 32, 557-564.

Wu, H., Zhao, C. and Liang, H. (2004). Comparison of linear, nonlinear and semiparametric mixed-effects models for estimating HIV dynamic parameters, Biom. J., 6, 233-245. 